Article

\title{
Detecting Emotions in English and Arabic Tweets
}

\author{
Tariq Ahmad ${ }^{1, *(\mathbb{D})}$, Allan Ramsay ${ }^{1}\left(\mathbb{D}\right.$ and Hanady Ahmed ${ }^{2}(\mathbb{D}$ \\ 1 School of Computer Science, The University of Manchester, Oxford Road, Manchester M13 9PL, UK; \\ allan.ramsay@manchester.ac.uk \\ 2 CAS, Arabic Department, Qatar University, Al Hala St, P.O. Box 2713 Doha, Qatar; hanadyma@qu.edu.qa \\ * Correspondence: tariq.ahmad@postgrad.manchester.ac.uk; Tel.: +44-161-275-2611
}

Received: 21 January 2019; Accepted: 28 February 2019; Published: 6 March 2019

\begin{abstract}
Assigning sentiment labels to documents is, at first sight, a standard multi-label classification task. Many approaches have been used for this task, but the current state-of-the-art solutions use deep neural networks (DNNs). As such, it seems likely that standard machine learning algorithms, such as these, will provide an effective approach. We describe an alternative approach, involving the use of probabilities to construct a weighted lexicon of sentiment terms, then modifying the lexicon and calculating optimal thresholds for each class. We show that this approach outperforms the use of DNNs and other standard algorithms. We believe that DNNs are not a universal panacea and that paying attention to the nature of the data that you are trying to learn from can be more important than trying out ever more powerful general purpose machine learning algorithms.
\end{abstract}

Keywords: sentiment mining; shallow learning; multi-emotion classification

\section{Introduction}

There are many ways to detect emotion. Quintana et al. [1] showed how to recognise emotion using the variability of heart rate. Nakasone et al. [2] used electromyography and skin conductance to determine emotion, and Busso et al. [3] recognised emotions using facial expressions and speech. This paper focuses on detecting emotions from documents.

According to recent research by Young et al. [4], deep learning methods are currently the state-of-the-art results in many domains. This trend was confirmed by the number of entries in the recent SemEval-2018 competition [5], where the winning entries in the English and Spanish [6,7] multi-classification competition all made use of neural networks. Indeed, many of the top-performing entries made use of a special kind of neural network known as "long short-term memory", either in conjunction with attention mechanisms [8,9], word embeddings [10,11] or convolutional neural networks [12,13].

The key features when trying to assign sentiment labels to documents are the words that they contain. Most approaches to this task involve the explicit or implicit construction of a sentiment lexicon. We explore two ways of constructing such a lexicon, either by explicitly counting the occurrences of words in documents that have been assigned a (possibly empty) set of labels drawn from a standard set of sentiment names or by building a deep neural network (DNN) with the set of words in the training set as input features and the sentiment names as output features. In the latter case, the lexicon is implicit in the weights that link the input layer, via the hidden layers, to the output layer. Such an implicit lexicon is often referred to as a word embedding; in essence, however, it is just a lexicon where each word is connected to a set of labels through a collection of arithmetical calculations.

Given that the task we are undertaking involves assigning a set of labels, we have to be careful about how we use DNNs. There are two obvious ways to proceed. (i) Given a set of features, it would be possible to train a set of classifiers, one per feature. Each classifier will return YES/NO for the 
feature on which it was trained. Taken together the set of classifiers can be seen as a single multi-label classifier. We refer to classifiers trained in this way as multi-DNNs. (ii) Train a single classifier with a node in the output layer for each feature and then accept every label for which the output-layer node had an excitation level above some threshold. We refer to this kind of classifier as single-DNN.

Emotion classification tasks have traditionally been tackled by obtaining a large corpus, constructing feature sets, pre-processing in sophisticated ways and then making use of any number of black box training algorithms $[14,15]$. Indeed, this approach is still prevalent currently $[6,7,16]$. We demonstrate that with a small-sized corpus and without using any black box algorithms, our results are comparable with systems that have been trained on millions (and sometimes billions) of tweets.

We show that the approach through explicitly constructing a lexicon substantially outperforms both of the DNN algorithms outlined above, and we speculate about why this should be so.

\section{Materials and Methods}

\subsection{Data Collection}

We used the data provided for the SemEval-2018 Task 1: Affect in Tweets task for English and Arabic. The organisers provided training, development and test datasets of tweets in Arabic and English. The tweets in the training and development datasets were each labelled with a (possibly empty) set of emotions. To create the English datasets, firstly, 50-100 terms were selected for each emotion. These were terms that were associated with the emotion, but at different intensity levels (e.g., for "angry" the terms "mad", "frustrated", "annoyed", "peeved", "irritated", "miffed", "fury" and similar terms were used). These were either obtained from Roget's Thesaurus or were nearest neighbours of the emotion words in a word-embedding space. Twitter was polled for the period June-July 2017 for tweets that included these query terms, and tweets were randomly selected for annotation. Finally, 100 tweets that had an emotion-word hashtag, emoticon or an emoji query term at the end were selected for each emotion, and the trailing query term was removed. Consequently, the final dataset also included tweets that contained no clear terms that indicated the emotion. The Arabic dataset was constructed in a similar fashion, but the English query terms were translated into Arabic using Google Translate, and EmoLex [17] was used to find words associated with the emotions. The authors describe how 17 million tweets were collected by polling Twitter for tweets that included emotion-related words such as "angry", "annoyed", "panic", "happy", "elated", "surprised", etc. The full list of query terms, for both languages, is available in their paper [18].

It is worth noting here that the Arabic tweets typically contained more words than the English ones. Arabic tweets had an average of 27 words, whereas for English tweets, it was 16. We believe that this reflects the fact that Arabic is typically written in a very condensed form, in which the marks corresponding to short vowels and other phonetically-relevant items (diacritics) are omitted. This is similar to the use of "textese" in English, wherein a text like "This message is heavily abbreviated" might be written as "This msg is holy abbroted". The omission of diacritics from written Arabic introduces more ambiguity than is the case for English written as textese; this makes Arabic tweets harder to analyse than English tweets. At the same time, it also means that it is possible for there to be significantly more words in an Arabic tweet than an English tweet. In a sense, this makes the task easier, since the more words there are, the more information there is about what the tweet says. As we will show, the results we obtained for the English and Arabic sets were extremely similar; hence, we believe these two effects cancelled each other out.

Having obtained tweets using the query terms, Mohammad et al. used a "somewhat generous" criteria when deciding which tweets to include in the dataset. Seven people were asked to annotate the tweets, and if at least two indicated that a certain emotion was inferred, then that emotion was chosen as one of the labels for the tweet.

It was surprising to see the relatively low annotator agreement levels; 0.48 for the Arabic dataset and 0.40 for the English dataset. These fall into the "moderate agreement" [19] band in 
the interpretations of Fleiss' kappa, indicating that the annotation was not a straightforward task due to its subjective nature.

Low annotator agreement is not uncommon. For example, Purver and Battersby [20] found very little agreement between the annotators when they manually labelled tweets with Ekmans [21] six emotions. Similarly, the work in [22-24] all reported low levels of inter-annotator agreement.

There were a number of interesting observations:

- There were more emotions than tweets in each dataset; this was to be expected since tweets can, and often do, contain multiple emotions.

- There were approximately the same number of average emotions per tweet across all four datasets; it is not clear whether this is by design or naturally occurring.

- The distributions of emotions across datasets from the same language were similar and, in some cases, (e.g., anger) were the same across all the datasets.

- Table 1 shows the breakdown of tweets by emotion. The table also shows that anger was the most popular emotion. Anger is an emotion that manifests itself by hostility towards someone or something. According to psychologists, anger can be a "substitute" emotion, meaning that people can make themselves angry so that they do not have to experience an even worse emotion such as pain. Fan et al. [25] collected 70 million tweets from Sina Weibo (a Chinese microblogging website) to examine how tweets that channelled specific emotions (joy, sadness, anger and disgust) influenced other people across the site. They found that "the most observable pattern was in the spread of angry tweets". This means that if a user sent an angry tweet, that anger emotion is likely to leak into the tweets of not only the followers who saw the tweet, but also their followers, and their followers' followers. They concluded that anger spreads faster than any other emotions on Twitter, and this could help explain why anger is the most popular emotion in the SemEval 2018 datasets. The next most popular emotion was sadness, followed closely by joy, possibly for similar reasons. At the opposite end of the scale are surprise and trust. This is likely because aside from the emotion words themselves, there are not many other words that convey these emotions. Furthermore, these are hardly emotions that one would see being shared amongst social media users.

The final datasets (the datasets are available from https: / competitions.codalab.org/competitions / 17751\#learn_the_details-datasets) consisted of a training set of around 6.8K English tweets (109K words) and $2.2 \mathrm{~K}$ Arabic ones (62K words) and development sets of around 886 tweets (13.9K words) and 586 tweets (15.8K words), respectively.

Table 1. SemEval-2018 tweets by emotion (percentages).

\begin{tabular}{cccccccccccr}
\hline Dataset & ang. & ant. & dis. & fea. & joy & lov. & opt. & pes. & sad. & sur. & tru. \\
\hline Arabic training & 17 & 4 & 8 & 6 & 12 & 12 & 12 & 10 & 16 & 1 & 2 \\
Arabic test & 16 & 4 & 8 & 7 & 12 & 12 & 12 & 9 & 16 & 1 & 3 \\
English training & 16 & 6 & 16 & 8 & 16 & 4 & 12 & 5 & 13 & 2 & 2 \\
English test & 15 & 6 & 15 & 6 & 18 & 5 & 14 & 5 & 12 & 2 & 2 \\
\hline
\end{tabular}

\subsection{The Task}

The task (named Task "E-c" in the competition) was to classify each tweet in the test set as "neutral or no emotion" or as one, or more, of eleven given emotions, \{anger, anticipation, disgust, fear, joy, love, optimism, pessimism, sadness, surprise and trust\}, that best represented the mental state of the tweeter.

\subsection{Algorithm}

Our weighted conditional probabilities (WCP) strategy was based on:

1. Using the tweets in the annotated training dataset to create word vectors. 
2. Using the word vectors to create a lexicon of tokens and conditional probabilities.

3. Transforming the probabilities into "scores".

4. Autocorrecting the lexicon by iteratively using a range of thresholds to remove unhelpful words.

5. Using the modified lexicon to calculate the threshold that achieved the best results for each emotion.

6. Classifying the test data using the modified lexicon and the best set of thresholds.

The outcome of this process was a trained model that could be used to classify the unseen, unannotated, tweets in the test dataset. Figure 1 shows an overview of the methodology architecture. Each stage is explained in further detail in this section.

Training

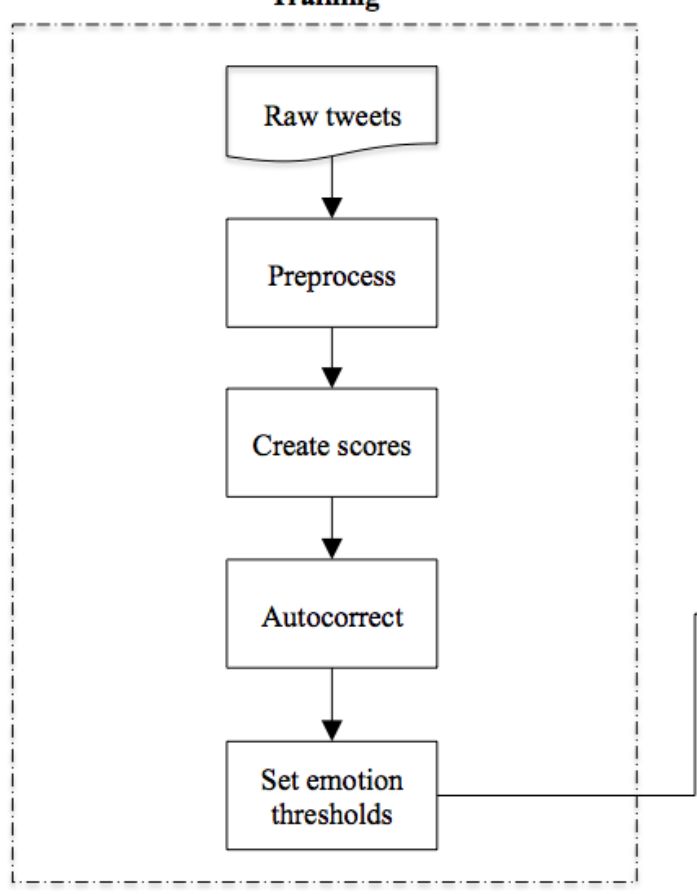

Testing

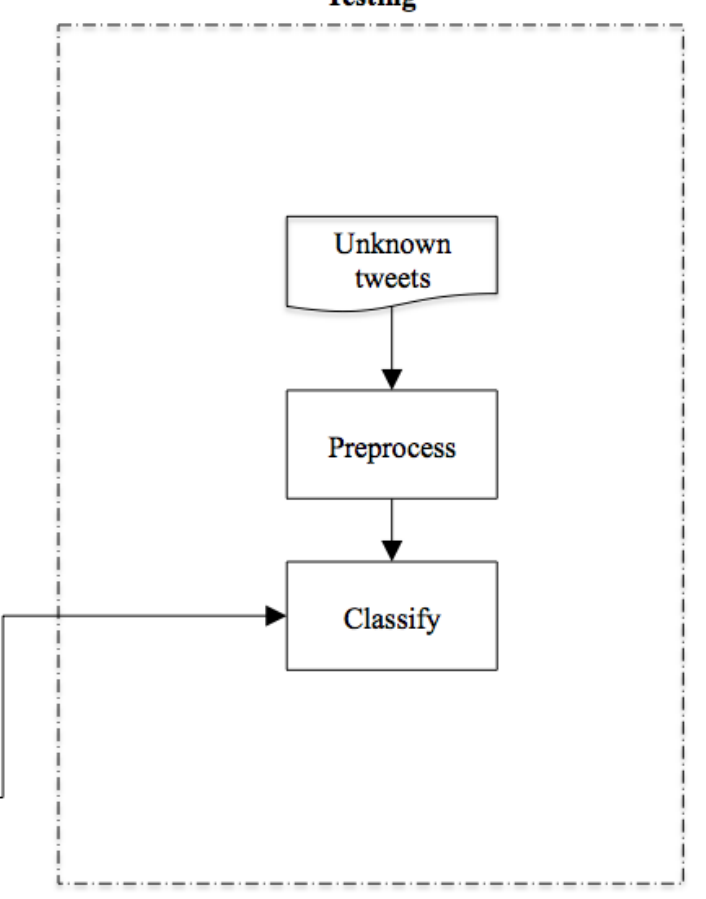

Figure 1. Architecture diagram.

\subsubsection{Preprocessing}

Preprocessing is an important and crucial step to obtaining good results; hence, each tweet was preprocessed, and a set of tokens and a count of each distinct token in the tweet were obtained. Tweets were preprocessed by lowercasing (English tweets only), removing Arabic and English punctuation, identifying and replacing emojis with emoji identifiers, expanding hashtags and then tokenising and stemming.

We believed that multi-word hashtags (e.g., \#better_world, \#late-night) also contained useful information. Consequently, to improve the quality of information in the tweet, hashtags were left unaltered, but a copy of the hashtag was taken and split into its constituent words ("expanded").

Two tokenisers were developed for use on both languages: one was based on the Natural Language Toolkit (NLTK) [26] and did not preserve hashtags, emoticons, punctuation and other content, and one was "tweet-friendly" because it preserved these items.

As the name suggests, emojis are used to convey emotions and hence were very significant for the current task. It was tricky to identify them, since the set is not fixed, and they also caused many technical problems due to their surrogate-pair nature. Hence, these were replaced with emoji identifiers (e.g., _45_). Contiguous instances of emojis were also separated out. This is because the individual emojis in a group of repeating unhappy face emojis should be recognised, and processed, as being the same emoji as a single unhappy face emoji. Usernames were also removed because we believed they were noise, since, by and large, they did not reappear in the test set, were not helpful and if left 
in would compromise the ability to detect useful information and would add, unnecessarily, to the computational load.

Up to this point, the steps for both languages were the same.

Stemming the words that appeared in a tweet was challenging, since the vocabulary used in tweets was very open and included numerous slang items and (deliberate and accidental) misspellings, so that stemmers that relied on a fixed lexicon (e.g., Madamira for Arabic [8]) did not work well on tweets.

Arabic and English tweets were tagged and stemmed in different ways.

\subsubsection{Arabic Tagging and Stemming}

Two techniques were developed to stem Arabic tweets, a "simple" method and a more complex method.

In the simple method, there was no explicit tagging stage. The token was simply firstly stemmed as a noun and then again as a verb using the stemmer developed specifically for Arabic tweets by Albogamy and Ramsay [27]. The shortest answer returned (in terms of the number of characters) was then taken as the answer. For example, when the Arabic word يدرسون (they study, present tense, masculine, plural) was stemmed as a noun, the result was يدرس (he studies, present tense, masculine, singular); when it was stemmed as a verb, the result was درس (he studied, past tense, masculine, singular). In this example, therefore, the verb form was taken as the correct stemmed form.

In the second method, Albogamy and Ramsay's tagger [28] was firstly used to establish the tag (noun, verb, etc.). This tag was then used to stem the word, again using the stemmer developed by Albogamy and Ramsay [27].

\subsubsection{English Tagging and Stemming}

Two similar techniques were also developed to stem English tweets. In the simple method, tweets were stemmed by, again, taking the shortest result from Morphy [29] when tokens were stemmed as nouns, verbs, adjectives and adverbs. Consider the following example (not from Morphy): the word "beautiful" has many different forms, such as beauty (noun), beautify (verb), beautiful (adjective) and beautifully (adverb). In this example, the noun would be taken as the correct stemmed form.

In the second method, the NLTK parts-of-speech tagger was used to generate the tags for the tokens. These tokens were then mapped to Morphy tokens, and Morphy was used to stem the word with the derived tag.

These preprocessed tokens were used to create counts of the number of tweets each token appeared in, and the reciprocal of each of these values was calculated to create an inverse document frequency (IDF) vector.

Common words such as "a" and " $\mathrm{i}$ " had scores close to 0 , whereas words that did not commonly appear had higher scores and were, thus, more important.

\subsubsection{From Probabilities to Scores}

Manual inspection of the distinct tokens showed that taking the top 2500 tokens appeared to be a good compromise between ignoring uncommon tokens and ensuring that the important tokens were captured. The most important tokens were extracted by, firstly, removing singletons (tokens that only occurred once in the dataset) and then simply taking the top 2500 tokens with the smallest IDF values (recall, IDF is small for commonly-used words and bigger for words that are used infrequently).

Ignoring uncommon tokens was important because, realistically, it could not be expected that place names, product names and brand names would be useful. Furthermore, since these words occurred very infrequently, it was unlikely to be possible to learn anything from them, and as they had little effect on the overall results, they only added extra unnecessary computational workload. Note that the words themselves remained present in the tweets; their scores were simply adjusted in the lexicon. 
Our approach was firstly to create probabilities and then to use these probabilities as a basis for constructing "scores". The probabilities were not used to construct scores for the individual emotions, but instead to construct scores relative to the other emotions. Constructing scores in this manner allowed us to make the observation that words such as "blessed" were much more significant for emotions such as joy, love and optimism than they were for emotions such as anger and anticipation and also then to take appropriate actions. Words that were insignificant had small scores, whereas words that were significant had large scores.

Lexicon

A base lexicon was created that contained every token in every tweet and a count of how often the token occurred against each of the emotions.

Tokens like "a" and "the" appeared many times across all emotions; consequently, they were of little importance. However, highly emotive tokens such as "rage" and "fury" appeared less frequently, but, crucially, appeared highly in negative emotions. A count of the number of tokens in each emotion was also obtained.

\section{Raw Conditional Probabilities}

For all tokens $T_{i}$ and all emotions $E_{j}$, the conditional probability $P\left(T_{i} \mid E_{j}\right)$ that a tweet that expresses the emotion $E_{j}$ will contain the token $T_{i}$ was calculated.

Normalisation

These probabilities were then normalised by dividing each probability by the sum of all the probabilities for the token. This step removed any knowledge of how common the emotion was, since we were just looking at the relative likelihoods of the word occurring with different emotions. For each token $T_{i}$, the average value $A\left(T_{i}\right)$ of the probabilities of that token over all emotions, i.e.,

$$
\sum_{j=1}^{k} P\left(T_{i} \mid E_{j}\right) / k
$$

was calculated and subtracted from each of the individual probabilities $P\left(T_{i} \mid E_{j}\right)$. This was a sort of local IDF step in that it had the effect of downplaying the significance of words that occurred equally frequently in tweets expressing different emotions. In other words, if a token was equally common for all emotions, then the token should not be important for any of then. Conversely, if a token was less than the overall average for a given emotion, then it should "vote" against that emotion.

Skew

Each conditional probability $P\left(W_{i} \mid E_{j}\right)$ was multiplied by the variance:

$$
\sqrt{\sum_{j=1}^{k}\left(P\left(T_{i} \mid E_{j}\right)-A\left(T_{i}\right)\right)^{2}} / k
$$

of the probabilities of that token for each emotion. The final scores for tokens like "the" were very close 0 , i.e., not particularly indicative towards any emotion. However, the scores for "fury" and "outrage" were large positive values for the emotions anger and disgust. These were the final scores used for classification. Every word in the vocabulary had a set of scores constructed in this manner. This step had the effect of increasing the significance of words that had very skewed distributions, i.e., when a token had an excessively scattered set of values, the difference between tokens that varied by a large amount was emphasised. A number of variations were tried, and we found that this worked best.

These initial steps produced a lexicon that linked words to emotions, as in Table 2. 
Table 2. Extracts from the English emotion lexicon.

\begin{tabular}{cccccccccccc}
\hline Token & ang. & ant. & dis. & fea. & joy & lov. & opt. & pes. & sad. & sur. & tru. \\
\hline admire & -0.457 & 0.438 & -0.516 & 2.640 & -0.287 & 2.133 & -1.533 & -3.402 & -3.402 & 7.787 & -3.402 \\
adorable & -6.112 & -6.112 & -6.112 & -6.112 & 7.245 & 41.354 & 0.299 & -6.112 & -6.112 & -6.112 & -6.112 \\
$\quad \ldots$ & & & & & & & & & & & \\
$\quad$ con & 24.690 & -5.457 & 19.868 & -5.457 & -0.902 & -5.457 & -5.457 & -5.457 & -5.457 & -5.457 & -5.457 \\
$\begin{array}{c}\text { inflame } \\
\text { outrage }\end{array}$ & 20.181 & -5.029 & 14.737 & 5.315 & -5.029 & -5.029 & -5.029 & -5.029 & -5.029 & -5.029 & -5.029 \\
$\quad \ldots$ & & -1.710 & 7.008 & -0.602 & -2.336 & -2.713 & -1.448 & -2.029 & -1.729 & -1.765 & -1.876 \\
$\begin{array}{c}\text { sick } \\
\ldots\end{array}$ & 1.623 & -2.167 & 1.526 & 1.748 & 0.195 & -3.219 & 0.365 & 0.734 & 5.634 & -3.219 & -3.219 \\
the & 0.018 & 0.074 & 0.004 & -0.006 & 0.001 & -0.368 & 0.104 & 0.189 & -0.082 & 0.023 & 0.043 \\
\hline
\end{tabular}

The lexicon showed that positive words scored highly for positive emotions and negative words scored highly for negative emotions. For example, Figure 2a,b shows the scores for "fury" and "outrage". These are words with negative connotations and, as expected, scored highly for the negative emotions anger and disgust. Essentially, depending on the other tokens in a tweet, it was highly likely that any tweet with these tokens would be classified as anger, disgust or both. Similarly, Figure 2c,d shows that the positive words "happy" and "joyful" scored highly not only for the expected emotions joy and love, but also surprisingly highly for optimism and trust. Neutral words were, naturally, not expected to contribute significantly, and this can be seen in Figure 2e where "the" scored near or equal to 0 for all emotions. The entry for "damn" is interesting since it is a word that can be used to express almost diametrically opposed emotions (e.g., "Damn I lost my keys and I forgot to get the garage opener" and "Damn gud \#premiere \#LethalWeapon...\#funny and \#serious"). Figure $2 \mathrm{f}$ shows that it made a positive contribution to a range of emotions, including anger and disgust, as well as making negative contributions to emotions such as fear and trust.

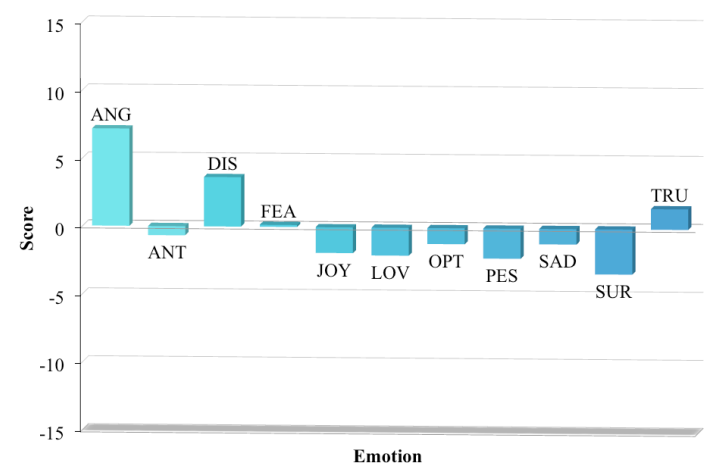

(a) Scores for "fury".

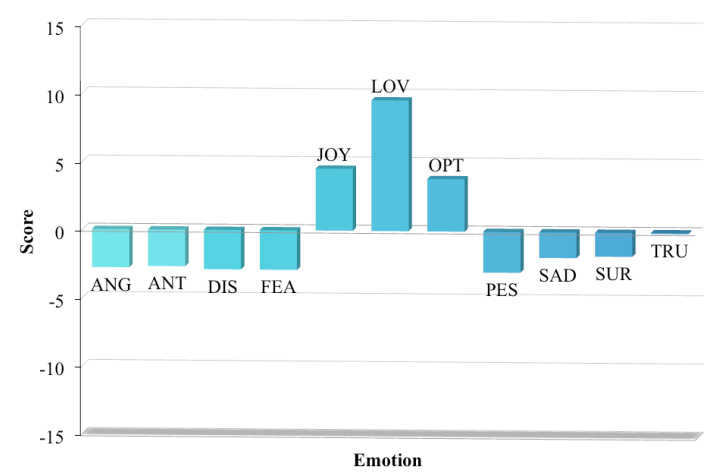

(c) Scores for "happy".

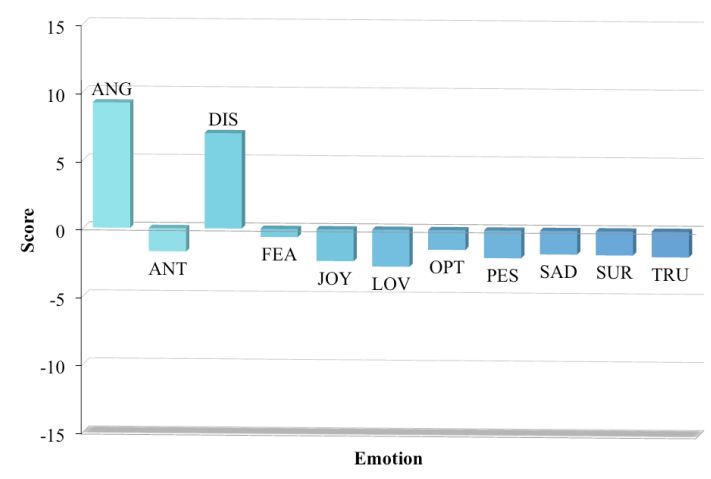

(b) Scores for "outrage".

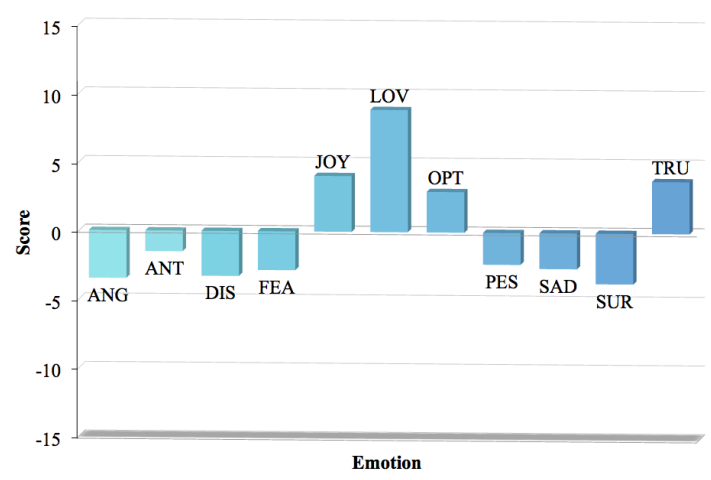

(d) Scores for "joyful".

Figure 2. Cont. 


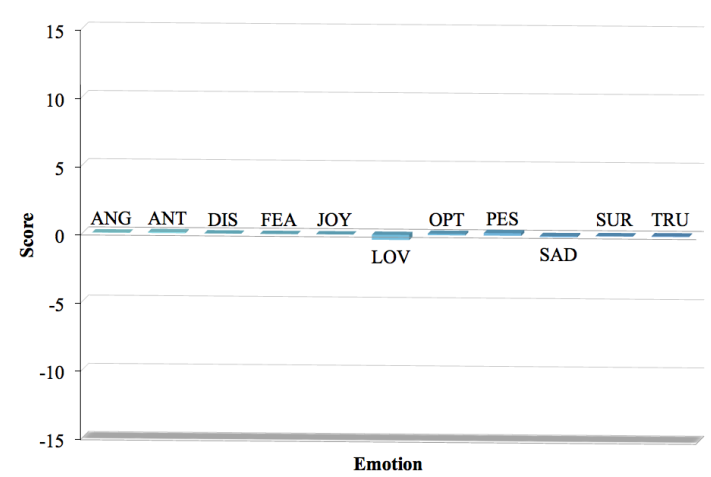

(e) Scores for "the".

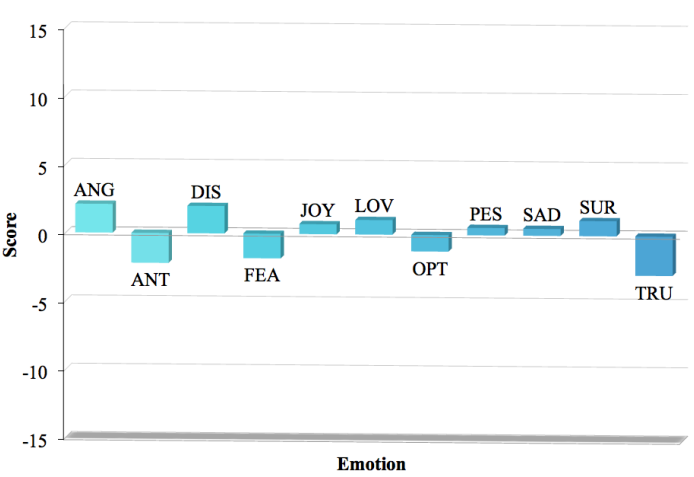

(f) Scores for "damn".

Figure 2. Graphs showing scores for words.

\subsubsection{Autocorrection}

Each token had an associated score for every emotion. This was a measure of how much evidence the token supplied for the emotion. However, there were situations where although the token appeared to provide evidence for an emotion, in reality, there were more tweets that contained the token that did not express the emotion.

Autocorrection removed these words from the lexicon. Each tweet was classified to determine the emotions it contained. A tweet was classified for each emotion by adding the lexicon scores for each token for each emotion, normalising and then comparing to a threshold $t$. If the score was less than $t$, then the tweet did not contain the emotion, otherwise it was classified as containing the emotion. This is where tokens voted "against" an emotion by contributing a negative value to the overall score and thus attempting to keep the overall score below the emotion threshold, hence preventing the tweet from being classified as that emotion. In exactly the same manner, tokens voted "for" an emotion by contributing high positive scores to the overall tweet score, thus pushing the score towards the emotion threshold and increasing the likelihood that the tweet would be classified as that emotion. As each tweet was classified, the predicted emotions were compared to the tweet gold standard (recall, the test set was also annotated with the tweet emotions). Every token in the lexicon was assigned a counter for each emotion, i.e., each token had 11 counters. If a tweet was classified correctly, the counter for every token in the tweet was incremented for each of the correctly-classified emotions. The counters were decremented if the tweet was classified incorrectly. When all the tweets were classified, these counters were examined, and for each token, if a counter was negative, this was evidence that the token was unhelpful in classifying tweets for that emotion, and its significance was downplayed in further calculations by allocating it a score of 0 for that emotion. This was an easy thing to do as the lexicon was explicit; it would have been much harder if the lexicon was encoded in a combination of weights, for example, as in a DNN. Using this technique, it was possible to remove tokens such as "celebrate" from incorrectly contributing to emotions such as fear.

\subsubsection{Thresholds}

A single fixed threshold across all emotions produced poor results because the raw data for each emotion were different and also because some emotions were easier to identify than others. Consequently, a range of thresholds was used to classify tweets on an emotion-by-emotion basis to generate an individual threshold for each emotion.

A varying threshold was used in the autocorrection stage to determine the words that were unhelpful because it was unclear what a sensible threshold was. Similarly, for classification, a varying threshold $t$ (from $0-1$ in steps of 0.1 ) was used to calculate the optimal threshold for each emotion.

For a given threshold, the modified lexicon was used to classify every tweet, one emotion at time. The overall Jaccard score for the test dataset was calculated for each threshold, and the best thresholds for each emotion were used for classification of unseen tweets. 
The thresholds were obtained by running the classifier on the original training data and choosing the optimal threshold for that data. This was a methodologically-sound way to make use of the data. Problems could have arisen if the training and test data were mixed, but care was taken not to do this.

Setting individual thresholds for each emotion reflected the fact that some emotions were more common than others, and hence, the algorithm needed to be more generous about predicting them (i.e., a lower threshold was required, and thus, lower tweet scores should be able to trigger classification as that emotion).

\subsubsection{Classification}

To obtain the best combination of lexicon and thresholds, an autocorrected lexicon was generated for each threshold $t 1$ from 1.0-0.0. Each of these lexicons was then used to classify the training tweets on an emotion-by-emotion basis for another threshold, $t 2$, between 1.0 and 0.0 . Each lexicon generated by using $t 1$ and each set of thresholds generated by using $t 2$ were then used to classify the development data. The pair that generated the best results was considered as the final model; hence, the final model consisted of a lexicon of token scores and a set of thresholds. This was then used to classify the unseen tweets, and these classifications were submitted as our entry to the SemEval-2018 competition.

Figure 3 shows the individual token scores, the values when they were summed and the sums after they were normalised for a sample tweet. The table also shows the optimal threshold for each emotion. The colour scale shows tokens that voted for an emotion (green), neutral tokens (yellow) and tokens that voted against an emotion (red). Each normalised score was compared to the optimal emotion threshold; if the score was greater than the threshold, then the conclusion was that the tweet contained that emotion. The figure shows that the high scoring tokens "blood" and "boil" were largely responsible for the tweet being classified as anger and disgust. The token "boil" also scored highly for sadness, but the negative score of "lord" for this emotion prevented the tweet from being classified as sadness. The tweet was also classified as trust; this was due to the large score for "lord" in the trust emotion. As it happens, this was incorrect, as the correct annotation for the tweet provided by the competition organisers was anger and disgust.

\begin{tabular}{|c|c|c|c|c|c|c|c|c|c|c|c|c|c|}
\hline & oh & lord & that & would & have & had & my & blood & boil & $\begin{array}{l}\text { normalised } \\
\text { sum }\end{array}$ & threshold & annotation & classified as \\
\hline ang. & 0.081 & -3.831 & 0.026 & -0.153 & -0.216 & 0.479 & -0.208 & 5.863 & 12.391 & 1.000 & 0.2 & 1 & 1 \\
\hline ant. & -1.243 & -3.289 & 0.320 & 1.332 & -0.023 & -0.177 & -0.485 & -0.617 & -0.702 & -0.338 & 0.4 & 0 & 0 \\
\hline dis. & 0.281 & -3.507 & 0.012 & -0.201 & -0.210 & 0.417 & -0.315 & 4.687 & 9.466 & 0.737 & 0.1 & 1 & 1 \\
\hline fea. & 0.769 & -1.402 & 0.092 & -0.051 & 0.479 & -0.291 & 0.257 & -2.220 & -4.154 & -0.452 & 0.8 & 0 & 0 \\
\hline joy & 0.235 & 1.892 & -0.096 & -0.460 & -0.427 & -0.168 & 0.013 & -2.188 & -2.754 & -0.274 & 0.0 & 0 & 0 \\
\hline lov. & 0.001 & -1.634 & -0.181 & -0.695 & -0.108 & -0.210 & 0.586 & -3.260 & -4.154 & -0.669 & 1.0 & 0 & 0 \\
\hline opt. & -0.294 & 6.098 & 0.181 & -0.428 & -0.596 & -0.302 & -0.294 & -1.973 & -2.474 & -0.006 & 0.0 & 0 & 0 \\
\hline pes. & 0.224 & -1.967 & 0.023 & 0.061 & 0.571 & -0.363 & 0.747 & 0.050 & -1.993 & -0.183 & 0.1 & 0 & 0 \\
\hline sad. & -0.032 & -3.298 & 0.022 & -0.144 & 0.290 & 0.679 & 0.809 & -1.297 & 2.679 & -0.020 & 0.3 & 0 & 0 \\
\hline sur. & 1.339 & -4.168 & -0.577 & -0.529 & 0.278 & 1.481 & -0.402 & 0.591 & -4.154 & -0.426 & 0.6 & $\mathbf{0}$ & 0 \\
\hline tru. & -1.359 & 15.106 & 0.177 & 1.269 & -0.038 & -1.545 & -0.707 & 0.363 & -4.154 & 0.631 & 0.0 & 0 & 1 \\
\hline
\end{tabular}

Figure 3. Sample tweet classification.

\subsection{Other Methods}

The task we are interested in is a multi-label classification. There are a number of standard algorithms that can be exploited for such tasks, either directly or by training a series of individual classifiers. We carried out a series of experiments with support vector machines (SVMs) and deep neural nets.

\subsubsection{Support Vector Machines}

The SemEval competition results included a number of baselines, one obtained by training an SVM on the distributed training data and one by making random choices. The SVM baseline was higher than anything we had managed to achieve using SVMs, and hence, we include it here for 
comparison with the other approaches. The SemEval scores were obtained on the competition test set, for which we do not have the gold standard labels. Our own results were better on the gold standard than on the development set, suggesting that in some sense, the gold standard test set was easier than the development set. In order to obtain a fair comparison of the various approaches, we included the results for WCP and the DNN-based approaches on the development set and for WCP and the SVM on the competition test set. These results are recorded in greyed out format in Table 3 to indicate that the SVM results were obtained from an external source.

Table 3. Scores on Arabic and English SemEval data. WCP, weighted conditional probabilities; DNN, deep neural network.

\begin{tabular}{|c|c|c|c|c|c|c|c|c|}
\hline & \multicolumn{4}{|c|}{ Arabic } & \multicolumn{4}{|c|}{ English } \\
\hline & Precision & Recall & F & Jaccard & Precision & Recall & F & Jaccard \\
\hline WCP & 0.620 & 0.632 & 0.626 & 0.455 & 0.589 & 0.658 & 0.622 & 0.451 \\
\hline single DNN & 0.601 & 0.537 & 0.567 & 0.396 & 0.624 & 0.488 & 0.587 & 0.416 \\
\hline multi-DNNs & 0.611 & 0.528 & 0.567 & 0.395 & 0.624 & 0.546 & 0.582 & 0.411 \\
\hline (WCP & & & & 0.484 & & & & $0.508)$ \\
\hline (SVM-unigrams & & & & 0.380 & & & & $0.442)$ \\
\hline
\end{tabular}

The SVM algorithm performed extremely poorly on the SemEval data, simply choosing the majority class in each case. We therefore concentrated on the DNN models. We used the SciKit Multilayerperceptron package, http:/ / scikit-learn.org/stable/modules/neural_networks_supervised.html, with sparse arrays for the DNN experiments. While TensorFlow can be faster to train if you can take advantage of multicore processing, the functionality of the two packages is very much the same, and it is unlikely that using TensorFlow would have significantly improved the performance of the DNN models.

\subsubsection{Deep Neural Nets}

There are two obvious ways of using DNNs for multi-label tasks. A single DNN is trained; the excitation levels of all the output nodes are inspected; and all the classes that surpass some specified threshold are chosen; or a DNN is trained for each class, and each is applied to the data being classified. It is worth noting that the second strategy implicitly assigns individual thresholds to sentiments as in the strategy outlined above.

We carried out both strategies on our data, with a DNN with three hidden layers, with the sizes of the three layers ranging from 50-400, from 20-80 and from 5-20, respectively. The numbers of nodes in the hidden layers made very little difference to the outcomes: the results in Table 3 were obtained with $(200,40,10)$ nodes in the hidden layers for the single DNN model and $(25,20,5)$ for the multi-DNN model for Arabic and $(200,80,10)$ for the single and $(25,20,5)$ for the multi-DNN model for English. Increasing the numbers of nodes in the layers beyond this led to overtraining and worse performance.

\subsection{Computing Resources}

The steps described in this section were performed using Python 2.7 on a MacBook Pro, 2.7-GHz Intel Core i5, 8 GB RAM.

\section{Results}

The results of these three strategies for the Arabic and English data from the SemEval task are given in Table 3. Mohammad et al. [5] included an SVM-based algorithm as a baseline, and we have included their Jaccard results for that on the SemEval test data in these tables (we do not have their precision, recall and F-measure results), since we were unable to obtain any useful results using SVMs, alongside the scores for WCP on the test data. 
For both datasets, the two DNN-algorithms had very similar scores. The multi-DNN versions, however, took much longer to train, since they required 11 distinct classifiers to be trained and tested. The DNNs seemed to outperform the SVM, but it should be noted that the SVM was tested on the SemEval test set, which may be easier than the development set. The ratios between WCP's scores on the development sets and the DNN's scores were lower than the ratios between WCP's scores on the test sets and the SVM's score (in plain English: WCP beat the SVM by more than it beat the DNNs; the DNNs were better than the SVM).

In general, the results for the Arabic and English were similar. Considering Arabic first, WCP performed well at identifying true positives. Some emotions (anger, joy, love, sadness) were more accurately identified than emotions such as anticipation, surprise and trust. Note, however, the extremely small number of training and test tweets for these emotions. True negatives were also identified well, for all emotions, with the lowest accuracy being 0.788 . Where true positives and true negatives were high, the opposite metrics, false negatives and false positives, were low. This was not true for the three difficult emotions anticipation, surprise and trust. Since WCP failed to identify these emotions adequately, the false positive measures were extremely high. Indeed, for trust,WCP failed to classify even one tweet correctly.

For the English dataset, WCP again generally performed well at identifying true positives apart from for the same emotions anticipation, surprise and trust. For surprise and trust, this may be again due to the small number of training tweets. However, for anticipation, the number of tweets could not have been a factor because fear also had a similar number of tweets, but WCP performed much better on fear. WCP also performed well at identifying true negatives even on the difficult emotions such as anticipation, surprise and trust. The accuracies were all above 0.7 and hence not particularly low. The lowest accuracies were on anger, disgust and optimism. These figures were evidence that the lexicon was doing its job in using high-scoring words to classify a tweet, but also at the same time preventing incorrect classifications. Consider the following tweet that was not annotated for anger, but was incorrectly classified as anger by WCP (i.e., a false positive):

\section{"If you build up resentment in silence are you really doing anyone any favors",}

When this tweet was processed by WCP, tokens such as "resentment" and "silence" had large scores for anger and thus contributed significantly in taking the score beyond the threshold for anger. It can be seen that these words can, reasonably, be considered as words that may be used to convey anger, e.g., in the tweets "Anger, resentment, and hatred are the destroyer of your fortune today." and "I'm going to get the weirdest thank you note-or worse-total silence and no acknowledgement.". The most common false positive misclassifications were joy being misclassified as optimism, anger being misclassified as disgust and sadness being misclassified as disgust. It is interesting to note that surprise-trust was the only mismatch that did not occur.

Although WCP performed well on some emotions, it was seen that there were some emotions that WCP found hard to classify with high accuracy. There were a number of possible reasons that may have caused WCP to perform poorly on these emotions including excessive emotion co-occurrences in tweets, not enough shared test and training tokens and a lack of emotion-bearing words.

\section{Discussion}

The experiments performed highlighted a number of factors that affected the performance of the classifier:

1. The effects of combining preprocessing steps such as lowercasing, removing punctuation and tokenising emojis were positive for the Arabic and the English datasets.

2. Expanding hashtags was a beneficial step for the English dataset, but detrimental in the Arabic dataset. This was because out of the 5448 distinct hashtags in the dataset, only 1168 (21\%) appeared five or more times. Consequently, this reduced their ability to have any meaningful impact on the classifier. 
3. Stemming using the tags from Albogamy and Ramsay's tagger almost always decreased classifier performance.

4. There were emotions (e.g., trust) that WCP found difficult to classify.

5. The sizes of the training and test datasets and the proportions of tweets for each emotion were significant factors in classifier performance. Increasing the training dataset size only had a positive effect if the new data were from a similar domain and were annotated in a similar fashion.

Although WCP does have its limitations as described above, we observed that it outperformed many of the SemEval-2018 competition entrants, with significantly lower computational complexity.

In order for WCP to be effective, various preprocessing steps were used. These steps were easier for English than for Arabic. For evaluation purposes, two vastly different languages were used. The structures of Arabic and English differ in many ways; Arabic is written from right to left; most of the Arabic letters join each other when writing; and there are many forms of words created by adding letters to the three-letter root word, each with a nuanced, specific meaning. Consequently, the English preprocessing was easier than that for the Arabic.

It was observed that simplistic tagging performed better than taggers trained on non-Twitter datasets. For English, standard Morphy stemming was used, but the Arabic stemmer was particularly effective because existing Arabic stemmers did not stem effectively. Although other entrants would also have had Arabic taggers and stemmers, we believe that the tools we used gave us a slight advantage.

The steps in WCP had the effect of boosting a token's score across all emotions if the difference between the mean across all emotions and the score for each emotion after the mean had been subtracted from it was high. This had the effect of double-counting, because subtracting the mean gave neutral tokens a score close to zero, and then, multiplying the actual score for each emotion by this variance emphasised the emotions for which the token was significant. This step simultaneously allocated words to emotions and gave extra weight to words that were much more important for one emotion than for the others. This had the effect of implicitly paying attention to both correlations and anti-correlations between emotions and allowed unhelpful tokens to be removed in the next stage.

Consider the word "rage". Figure 4 shows how the probabilities evolved into scores. The scores for the token started as probabilities and were very similar to each other. They remained closely clustered during normalisation and also the step where the average was subtracted from the normalised value. It was only when the values were skewed that the scores started to become distinguishable from each other in a meaningful fashion.

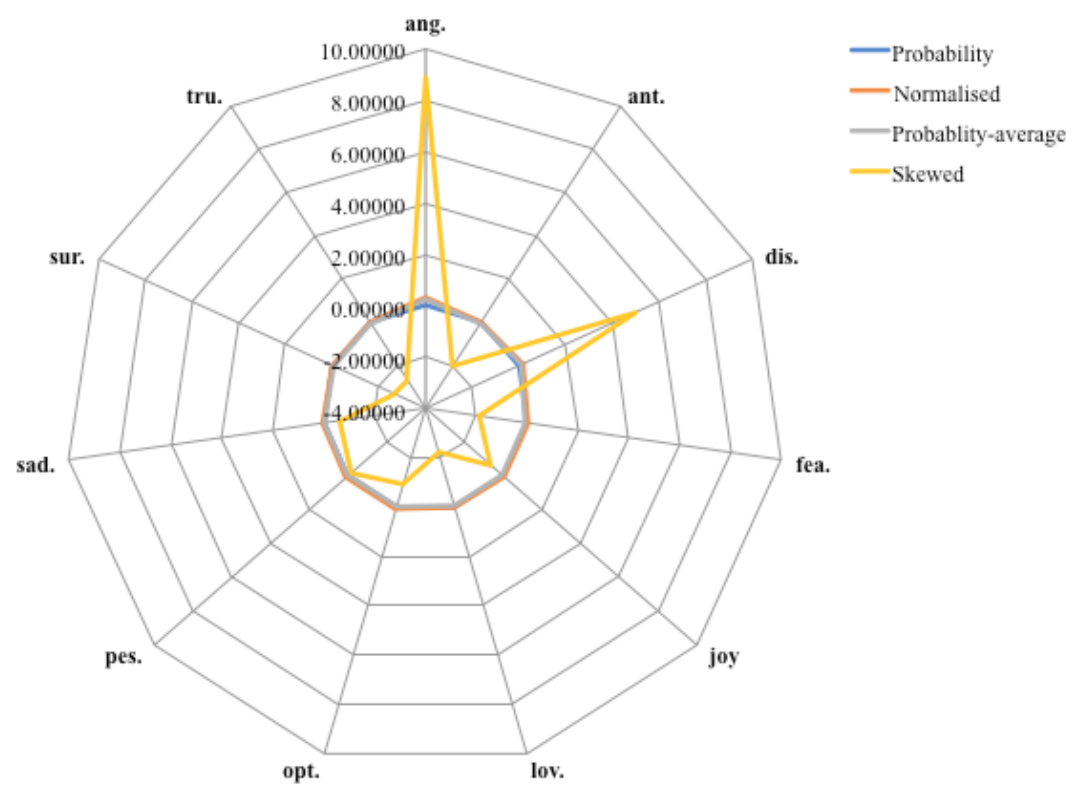

Figure 4. Evolution of "rage" from probability to score. 
Figures 5 and 6 show the number of tokens that were removed from each emotion at each threshold during the autocorrection stage for the Arabic and English datasets (the colour scale goes from green to red with green for the smallest values and red for the largest values). For both datasets, it can be seen that as the threshold decreased, more tokens were removed by the autocorrection process. This is because as the threshold decreased, a smaller and smaller score was enough to classify the tweet as an emotion; this led to more false classifications, and thus, more tokens were removed. Generally, the pure emotions were the least autocorrected. This is indicative of strong unambiguous words being used in these tweets. The tweets that WCP had difficulty with (anticipation, surprise and trust) had the highest numbers of autocorrections. This corresponds with the earlier findings that these tweets contained the highest numbers of multiple emotions, and thus, it was more likely that tokens would appear in other correctly-classified tweets, hence invoking autocorrection.

\begin{tabular}{l|cccccccccccc}
\hline $\mathbf{t}$ & ang. & ant. & dis. & fea. & joy & lov. & opt. & pes. & sad. & sur. & tru. & \\
\hline $\mathbf{1}$ & 8 & 20 & 6 & 12 & 8 & 3 & 4 & 12 & 10 & 70 & 20 & 173 \\
$\mathbf{0 . 9}$ & 7 & 27 & 11 & 11 & 10 & 2 & 6 & 12 & 13 & 79 & 28 & 206 \\
$\mathbf{0 . 8}$ & 15 & 23 & 15 & 11 & 5 & 4 & 8 & 20 & 11 & 86 & 35 & 233 \\
$\mathbf{0 . 7}$ & 11 & 31 & 26 & 13 & 4 & 6 & 5 & 15 & 12 & 103 & 46 & 272 \\
$\mathbf{0 . 6}$ & 7 & 51 & 31 & 19 & 4 & 7 & 6 & 17 & 17 & 116 & 61 & 336 \\
$\mathbf{0 . 5}$ & 8 & 53 & 36 & 29 & 3 & 7 & 8 & 27 & 15 & 133 & 90 & 409 \\
$\mathbf{0 . 4}$ & 5 & 75 & 56 & 45 & 5 & 17 & 14 & 35 & 16 & 152 & 131 & 551 \\
$\mathbf{0 . 3}$ & 4 & 126 & 85 & 83 & 3 & 24 & 16 & 61 & 20 & 167 & 196 & 785 \\
$\mathbf{0 . 2}$ & 7 & 212 & 114 & 119 & 20 & 35 & 25 & 131 & 28 & 189 & 281 & 1161 \\
$\mathbf{0 . 1}$ & 11 & 309 & 175 & 199 & 30 & 61 & 43 & 250 & 47 & 199 & 384 & 1708 \\
$\mathbf{0}$ & 26 & 461 & 296 & 202 & 44 & 90 & 83 & 419 & 77 & 217 & 483 & 2398 \\
& 109 & 1388 & 851 & 743 & 136 & 256 & 218 & 999 & 266 & 1511 & 1755 &
\end{tabular}

Figure 5. Heat-map showing autocorrection of tokens from each emotion in the Arabic dataset.

\begin{tabular}{l|cccccccccccc}
\hline $\mathbf{t}$ & ang. & ant. & dis. & fea. & joy & lov. & opt. & pes. & sad. & sur. & tru. & \\
\hline $\mathbf{1}$ & 14 & 131 & 21 & 27 & 9 & 109 & 20 & 184 & 35 & 311 & 393 & 1254 \\
$\mathbf{0 . 9}$ & 12 & 149 & 14 & 26 & 13 & 139 & 27 & 262 & 39 & 343 & 417 & 1441 \\
$\mathbf{0 . 8}$ & 14 & 161 & 13 & 29 & 11 & 158 & 28 & 281 & 38 & 387 & 451 & 1571 \\
$\mathbf{0 . 7}$ & 14 & 160 & 15 & 31 & 22 & 191 & 38 & 333 & 37 & 435 & 494 & 1770 \\
$\mathbf{0 . 6}$ & 16 & 175 & 15 & 41 & 21 & 227 & 35 & 398 & 32 & 494 & 521 & 1975 \\
$\mathbf{0 . 5}$ & 21 & 190 & 25 & 52 & 18 & 278 & 39 & 488 & 50 & 535 & 566 & 2262 \\
$\mathbf{0 . 4}$ & 21 & 214 & 23 & 60 & 14 & 319 & 40 & 556 & 60 & 594 & 609 & 2510 \\
$\mathbf{0 . 3}$ & 23 & 281 & 28 & 112 & 15 & 385 & 38 & 632 & 85 & 646 & 669 & 2914 \\
$\mathbf{0 . 2}$ & 26 & 430 & 36 & 168 & 11 & 434 & 36 & 731 & 125 & 698 & 714 & 3409 \\
$\mathbf{0 . 1}$ & 37 & 545 & 51 & 269 & 15 & 506 & 58 & 807 & 213 & 748 & 756 & 4005 \\
$\mathbf{0}$ & 51 & 680 & 72 & 431 & 22 & 572 & 89 & 897 & 337 & 783 & 797 & 4731 \\
& 249 & 3116 & 313 & 1246 & 171 & 3318 & 448 & 5569 & 1051 & 5974 & 6387 &
\end{tabular}

Figure 6. Heat-map showing autocorrection of tokens from each emotion in the English dataset.

At the largest threshold, 1.0, the least number of tokens were autocorrected. Autocorrection involved scoring and classifying tweets. Recall that one of the steps in scoring a tweet was to divide the scores for each emotion by the maximum emotion score. This process, by definition, set one of the emotion scores to 1.0. At the largest threshold, therefore, there could only be one emotion classified correctly; in effect, WCP reverted to a single-emotion classifier for this threshold. Hence, the largest threshold is perhaps of little use and should be discarded for autocorrection.

Tokens that appeared in multiple tweets labelled with multiple emotions were more likely to be removed due to the WCP autocorrection process. In other words, the more different emotions a token appeared against, the more likely it was to be removed.

Note that the autocorrection was only applied to true positives and false positives, i.e., when the tweet was annotated as having an emotion by the annotators. Only when a tweet had been annotated 
as having an emotion could autocorrection decide if it was useful or not; hence, tokens for tweets that had not been marked for an emotion were not used for autocorrection purposes.

Many tokens that were removed had no relationship to the emotion they were removed from and were removed only on the basis of being seen in a number of tweets that just happened to be misclassified, marginally, more often than classified correctly. For example, tokens such as "silver", "mope" and "drink" (anger), "address", "year" and "million" (fear), "buzz", "older" and "jersey" (joy) and "son", "inside" and "roll" (sadness) were all autocorrected on this basis.

Generally, those tokens that were removed that had much higher negative counts were more non-relevant to the emotion in the sense that one could easily imagine that they would not be useful in the emotions from which they were removed; for example, "horror", "revenge" and "grudge" (anticipation), "gloomy", "haunt" and "sadly" (love) and "nightmare", "panic" and "terrible" (pessimism). These tokens with much higher autocorrection counter values were predominantly in the non-pure emotions that were difficult to classify. This indicates the difficulty of the exercise, that even after downplaying tokens that were unhelpful, it was still difficult to classify these tweets. It was also seen that some autocorrected tokens had multiple meanings (e.g., "cool", "wicked", "bad", "gay"). These were tokens that originally meant something completely different (and sometimes opposite in emotion) to how they are used today.

Autocorrecting tokens had two effects:

1. Decreased the likelihood that tweets containing autocorrected tokens would be incorrectly classified.

2. Increased the likelihood that genuine tweets containing autocorrected tokens would be correctly classified.

Since the autocorrected tokens had been identified as being detrimental, the overall effect was that this increased the accuracy of the classifier.

It is important to note that this reclassification was carried out on the original training data. This was methodologically sound as the training data was not used for testing; it was simply reused as part of the overall training process. Experiments were performed where a portion of the training data was set aside for this purpose, but it turned out to be more effective to reuse the full set. It was obviously more important to have as much data as possible for this purpose than to keep the training and retraining data separate. This approach of autocorrecting was based on the suggestion of Brills [30] that one should attempt to learn from one's own mistakes.

A limitation of autocorrecting, however, is that there were tokens that were incorrectly autocorrected that could, conceivably, have been useful in the emotion in which they were autocorrected; for example, "intimidate", "defend" and "war" (fear) "cheerfulness", "cuddle" and "joyous" (love) and "together", "grateful" and "assistance" (trust). Autocorrection for tokens such as these was carried out purely on the basis that they appeared in more non-useful tweets. More training data may have rectified this issue as it could have been expected that there would have been more instances of the correct use of the tokens, thus preventing them from being autocorrected. However, even this may not have fully rectified the problem because there were tokens that had large counts indicating that, regardless of what one might believe, they genuinely were not helpful to the emotion, e.g., "nervous" ( -26 , anticipation), "anxiety" (-47, pessimism) and "love" ( -78, trust $)$.

On the whole, the autocorrection process was beneficial, improving the Jaccard score on the Arabic dataset from 0.342 to 0.370 and on the English dataset from 0.401 to 0.431 .

Experiments were performed running autocorrection multiple times, but it was found that very few words were removed after the first iteration. One possible explanation for this may be because the actual numbers of tokens that were removed was quite small: 1\% for Arabic and 5\% for English.

Choosing a threshold above which a new tweet was classified as an emotion vs. a non-emotion was an important step. The raw data for each emotion were different, and hence, a single fixed threshold across all emotions produced poor results. 
This step was implicitly carried out by the multi-DNN models: when a DNN was used as a classifier, i.e., when there were two output nodes, one for YES and one for NO, it calculated the optimal excitation level for the YES node. Thus, when a set of DNNs was used for multi-classification, each one had its own threshold. For the multi-DNN, it is likely that a single threshold was chosen for all the output nodes.

Figures 7 and 8 show the results for the Arabic and English datasets when WCP was used with a number of fixed thresholds compared to when a varying threshold was allowed, as well as the best thresholds for each emotion as determined by WCP. It was clear that WCP performed better on some emotions with higher thresholds and on others with lower thresholds. The " 2 " column indicates the threshold used, followed by the Jaccard score for each emotion at that threshold and the overall Jaccard score for all the tweets in the last column. The last row in the table shows the best Jaccard scores for each emotion and the overall Jaccard score. The black line shows the best thresholds for each emotion. The results for both datasets shared a number of characteristics. WCP selected lower thresholds for good performance on anger and anticipation. However, for disgust, fear, joy, love and optimism, the same threshold was selected for the Arabic dataset, whereas for the English dataset, the thresholds were variable. The last four emotions showed a clear pattern: pessimism and sadness required low thresholds; surprise needed a threshold higher than both of these; and trust needed a small threshold for good performance.

\begin{tabular}{|c|c|c|c|c|c|c|c|c|c|c|c|c|}
\hline$t 2$ & ang. & ant. & dis. & fea. & joy & lov. & opt. & pes. & sad. & sur. & tru. & Jaccard \\
\hline 1 & 0.343 & 0.153 & 0.559 & 0.741 & 0.449 & 0.330 & 0.356 & 0.231 & 0.432 & \multirow{3}{*}{$\AA^{0} \mathbb{2}^{65}$} & 0.339 & 0.278 \\
\hline 0.9 & 0.376 & 0.158 & 0.604 & 0.751 & 0.574 & 0.473 & 0.463 & 0.266 & 0.483 & & 0.385 & 0.325 \\
\hline 0.8 & 0.427 & 0.187 & 0.626 & 0.753 & 0.693 & 0.586 & 0.590 & 0.293 & 0.523 & & 0.426 & 0.356 \\
\hline 0.7 & 0.495 & 0.224 & 0.643 & 0.770 & 0.740 & 0.699 & 0.663 & 0.342 & 0.557 & 0.255 & 0.426 & 0.390 \\
\hline 0.6 & 0.579 & 0.229 & 0.663 & 0.769 & 0.784 & 0.736 & 0.716 & 0.409 & 0.591 & 0.255 & 0.451 & 0.417 \\
\hline 0.5 & 0.656 & 0.252 & 0.605 & 0.779 & 0.799 & 0.797 & 0.739 & 0.463 & 0.639 & 0.255 & 0.451 & 0.433 \\
\hline 0.4 & 0.720 & 0.308 & d. 657 & 0.775 & 0.795 & 0.776 & 0.738 & 0.516 & 0.668 & 0.250 & 0.463 & 0.435 \\
\hline 0.3 & 0.768 & 0.341 & 0.644 & 0.762 & 0.776 & 0.757 & 0.719 & 0.565 & $0.7 / 2$ & 0.245 & 0.480 & 0.439 \\
\hline 0.2 & 0.789 & 0.37 & 0.633 & 0.759 & 0.749 & 0.729 & 0.711 & 0.622 & لt 13 & 0.236 & d496 & 0.442 \\
\hline 0.1 & 0.779 & & 0.599 & 0.704 & 0.716 & 0.681 & 0.665 & & 0.702 & 0.232 & 0.504 & 0.449 \\
\hline 0 & 0.742 & $D, d 20$ & 0.535 & 0.649 & 0.680 & 0.630 & 0.605 & 0.586 & 0.637 & 0.220 & 0.483 & 0.444 \\
\hline * & 0.789 & 0.420 & 0.663 & 0.779 & 0.799 & 0.777 & 0.739 & 0.629 & 0.713 & 0.265 & 0.504 & 0.455 \\
\hline
\end{tabular}

Figure 7. Heat-map showing results of different thresholds for the Arabic dataset.

\begin{tabular}{|c|c|c|c|c|c|c|c|c|c|c|c|c|}
\hline $\mathrm{t} 2$ & ang. & ant. & dis. & fea. & joy & lov. & opt. & pes. & sad. & sur. & tru. & Jaccard \\
\hline 1 & 0.444 & 0.311 & 0.178 & 0.598 & 0.212 & $0.4^{81}$ & 0.236 & 0.148 & 0.364 & 0.271 & 0.097 & 0.227 \\
\hline 0.9 & 0.519 & 0.321 & 0.292 & 0.605 & 0.247 & $0 / 4 t 5$ & 0.268 & 0.170 & 0.392 & 0.272 & 0.102 & 0.256 \\
\hline 0.8 & 0.567 & 0.337 & 0.417 & $0.91^{5}$ & 0.280 & 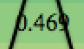 & 0.299 & 0.201 & 0.428 & 0.273 & 0.128 & 0.281 \\
\hline 0.7 & 0.590 & 0.347 & 0.504 & ofde & 0.316 & 0.463 & 0.326 & 0.228 & 0.458 & 0.285 & 0.137 & 0.309 \\
\hline 0.6 & 0.609 & 0.349 & 0.534 & 6.602 & 0.359 & 0.455 & 0.367 & 0.254 & 0.485 & 02 & 0.151 & 0.332 \\
\hline 0.5 & 0.623 & 0.358 & 0.560 & 0.595 & 0.423 & 0.440 & 0.405 & 0.276 & 0.500 & & 0.154 & 0.351 \\
\hline 0.4 & 0.640 & 6 & 0.578 & 0.571 & 0.490 & 0.427 & 0.443 & 0.293 & 0.513 & 0.285 & 0.163 & 0.370 \\
\hline 0.3 & 0.647 & & 0.595 & 0.544 & 0.558 & 0.408 & 0.485 & 0.301 & & 0.286 & 0.175 & 0.398 \\
\hline 0.2 & 0.649 & 0.359 & 500 & 0.517 & 0.624 & 0.389 & p.521 & 0.01 & 0.520 & 0.283 & 0.190 & 0.424 \\
\hline 0.1 & 0.649 & 0.359 & 0.665 & 0.489 & 6671 & 0.364 & 0,540 & & 0.508 & 0.290 & d 184 & 0.431 \\
\hline 0 & 0.634 & 0.338 & 0.597 & 0.448 & 0.673 & 0.346 & & 0.312 & 0.481 & 0.281 & 0.202 & 0.427 \\
\hline * & 0.649 & 0.366 & 0.605 & 0.615 & 0.673 & 0.481 & 0.545 & 0.314 & 0.521 & 0.291 & 0.202 & 0.456 \\
\hline
\end{tabular}

Figure 8. Heat-map showing results of different thresholds for the English dataset.

It is interesting to note the scores at these thresholds (recall that although the thresholds were important, the aim was to maximise the scores). WCP scored highly for anger at the low thresholds regardless of the dataset. However, this was the only correlation between the two datasets. For every other emotion, both datasets behaved differently. Some emotions needed high thresholds, but still 
scored poorly (e.g., surprise in the Arabic dataset); some emotions had low thresholds, but scored poorly (e.g., trust in the English dataset); some emotions had high thresholds and scored highly (e.g., fear in the English dataset); and some emotions had low thresholds and scored highly (e.g., pessimism in the Arabic dataset). This highlights that there were general similarities, but also key differences between the languages and how they were used to convey emotion in tweets.

For both datasets, WCP performed better at lower thresholds for anger. This reflected the fact that there were many strong words that were highly indicative of anger alone, e.g., "fuming", "inflame", "pissed", "scorn", "furious" and "rage". Words used to convey anger in the Arabic dataset were, perhaps, indicative of the current situation in certain Middle Eastern countries: الحوثيين ("Houthis"), الحوثيين ("irritation"), اعتداء ("military")assault"). Both sets of words were indicative of anger, and consequently, obtaining a high score was achieved more easily than for some of the other emotions. However, at the higher thresholds, the Jaccard score decreased because anger was confused with the other emotions, e.g., disgust. A small threshold was clearly not the only criteria for a good score, as can be seen in both datasets, where the best Jaccard for anger was much higher than the best Jaccard for anticipation, although both had low thresholds. It is interesting to see why the best thresholds were low for some emotions and high for others.

Higher scoring tokens for an emotion were indicative of strong emotion-bearing words for that emotion. Tweets that were classified as an emotion due to being strongly indicative towards an emotion and contained highly emotive words for that emotion remained classified as that emotion, regardless of the threshold. For example, consider the tweet: "Bloody parking ticket_145_217_\#fuming", where _145_represents the UNAMUSED FACE emoji and _217_represents the MONEY WITH WINGS emoji (because this was only used in this one tweet and was removed as a singleton, it had a score of zero). Due to the presence of "\#fuming" and "fume" (and their high scores), the normalised score for anger was 1.0 (i.e., the largest possible value). Consequently, this tweet was always classified as anger, regardless of the threshold.

However, this was not always the case, especially for tweets that contained words that were less emotive or could also be used for other emotions. For example, consider the tweet "brb going to sulk in bed until friday". For anger, the tweet only scored 0.177, largely on the basis of the token "sulk". Consequently, this tweet would only be classified as anger at the 0 and 0.1 thresholds. Beyond the 0.1 threshold, it would be classified as a false negative for anger, although it may be classified correctly for some other emotion.

Every emotion had a different optimal threshold; we believe this is due to the fact that different tokens were important for different emotions and also because there were different numbers of training tweets for each emotion. If there were more training tweets for trust, for example, there may have been more words specifically used for trust, and this may have led to more higher scoring tokens for trust.

Every emotion had tokens that scored highly and tokens that scored poorly. However, recall that for tokens that did not occur in some emotion (e.g., "furious" did not appear in any trust tweets), these low scores were always large and negative (e.g., -7). For example, recall that trust had the least number of training tweets. Consequently, for the trust emotion, the lexicon contained relatively few tokens that genuinely came from trust tweets, but many tokens for trust with large negative scores that came from other emotions, which would vote against trust. Consequently, according to Figures 7 and 8 , for a tweet to be classified as trust, it merely had to achieve a score large enough to exceed the minimum threshold. Even with this minimal threshold, the Jaccard score for trust was reasonable; however, this may be due to the small number of test tweets.

The higher the token scores were for an emotion, the more likely that tweets with those tokens would generate high normalised tweet scores (i.e., closer to the maximum threshold of 1.0). This made these scores larger than many of the (lower) thresholds, and consequently, the likelihood of the tweet being classified as that emotion was increased. In other words, emotions containing tokens with high scores tended to be easier to classify, and the Jaccard scores for these emotions tended to be higher. However, as seen in the tweet about "sulking in bed until Friday", not all angry tweets were 
straightforward to classify, and due to these tweets, as the threshold got higher, more and more angry tweets were classified as false negatives.

Although the final Jaccard scores were similar for both datasets, it is interesting to see that the emotive words used in the datasets were very different. It was observed that Arabs tend to use hashtags infrequently in tweets and that many of the top tokens in the Arabic dataset were referring to the situation in Yemen ("Houthis", "victory", "our land"). In general, it was difficult to draw concrete conclusions from the results because the Arabic dataset was small.

The top Arabic dataset tokens also scored substantially higher than their English counterparts. It is important to clarify that high scores did not indicate that a token appeared many times in an emotion or in a dataset, rather it was an indication of the importance of the token to an emotion relative to the other emotions. Consider the token الانتصار ("the victory"). This was the highest scoring token for trust in the Arabic dataset. However, this token appeared in only five tweets throughout this dataset, three of which were classified as trust:

"Experience killed mercenaries of Taiz with a weapon and I killed the mercenaries of Twitter. Praise be to God for these victories. Hehe"

"The feeling of victory"

"Some fans were surprised about the amount of frustration inside them, trust your team and let the predestination does as it please. Hala Madrid! after defeat comes victory"

The token also appeared twice in tweets classified as optimism. Thus, the probability for the token was greater for trust than it was for optimism. This difference led to WCP ultimately calculating a high score for the token for trust. In other words, large scores were generated on the basis of a small number of tokens; these tokens then went on to contribute over-excessively to tweets being classified as trust.

This step had a reasonable impact on the performance of WCP, since it implicitly weighed up the likelihood of a given emotion occurring at all, as well as being sensitive to the fact that different emotions may be expressed in different ways.

Separate thresholds for each emotion increased the overall scores from 0.370 to 0.452 on the Arabic dataset and from 0.431 to 0.455 on the English dataset.

These results show that this process, while clearly helpful, was not a major contributor to the difference between WCPs' performance on the target data and the multi-DNNs' performance, since the multi-DNNs also had similar processes. However, the final results on the two datasets were extremely similar, thus confirming the robustness and adaptability of WCP.

The key elements of WCP, autocorrection and thresholding, showed that with these in place, significant performance gains were achieved. This is consistent with the initial intuitions that allowing the algorithm to drop unhelpful words and that a single threshold for all emotions would not be helpful resulted in significant performance gains. We also observed that certain emotions often presented together in tweets as pairs. For example, joy and optimism often presented together. We believe that this also has an effect on the results.

It was noted that WCP performed poorly on the trust emotion. However, only $2 \%$ of the tweets in the datasets were labelled as trust. This was an extremely modest amount of tweets to learn from and may account for the low scores. However, the surprise emotion had an even smaller number of tweets, yet WCP managed to perform at least on par with some of the other emotions. This suggested something different about the tokens used in the trust and the surprise tweets. This difference in performance may be due to the differences in the numbers of tweets that have multiple emotions. For example, trust had almost three-times as many tweets that had 2, 3 and 4 emotions than surprise. It was also noticeable that the English datasets seemed easier to classify.

It is possible that the annotation process also played a part in shaping the results. The crowdsourcing nature of the annotation left much scope for judgement; hence, datasets constructed in this way are more difficult to classify due to noise and misclassifications. 
WCP was evaluated on the Arabic and English datasets provided for the task by the competition organisers, where it ranked second for the Arabic dataset (out of 14 entries) and $12^{\text {th }}$ for the English dataset (out of 35 entries). In the Arabic competition, WCP came second to Badaro et al. [31]. Badaro et al. presented a system called "EMA" (emotion mining for Arabic), reporting that linear SVC (support vector classifier) in conjunction with word embeddings gave the best performance. These word embeddings were obtained from AraVec [32], a word embedding model that was built from tweets, web pages and Wikipedia. More than three billion tokens were used to build the models. We believe that, in this context, to be outperformed by only 0.005 serves to highlight the effectiveness of our approach. In the English competition, the entries that outperformed us were either based on neural networks $[6,9-11,13,33]$ or word embeddings $[7,12,34]$, with a number of these also making use of external lexicons. The problem with using lexicons is that lexicons, stop-word lists and resources of this type are not always available for all languages. Furthermore, the language on Twitter is continuously evolving, and from this point of view WCP, has the advantage that it can be retrained quickly and easily.

\section{Conclusions}

In the work described here, we have shown that designing an algorithm that is targeted at the specific task under investigation (assignment of sentiment labels to Arabic and English tweets in the current study) can be more effective than just applying a standard general-purpose tool.

It may be, of course, that such algorithms have parameters that can be tweaked to improve their performance, but randomly tweaking parameters is a time-consuming and unilluminating activity. We believe that it is, in at least some cases, better to think about the nature of the problem and design an algorithm that works for it than to trust in the effectiveness of some pre-built black-box learning algorithm.

In summary, it has been demonstrated that the WCP approach of explicitly constructing a lexicon substantially outperforms both DNNs and SVM. This new algorithm also excelled at the internationally-recognised competition SemEval-2018.

Author Contributions: Conceptualization, T.A.,A.R. and H.A.; methodology, T.A. and A.R.; software, T.A. and A.R.; validation, T.A. and A.R.; formal analysis, T.A. and A.R.; investigation, T.A.; resources, A.R.; data curation, T.A.; writing, original draft preparation, T.A. and A.R.; writing, review and editing, T.A.; visualization, T.A.; supervision, A.R.; project administration, H.A.; funding acquisition, H.A.

Funding: This publication was made possible by the NPRP award (NPRP 7-1334-6-039 PR3) from the Qatar National Research Fund (a member of The Qatar Foundation). The statements made herein are solely the responsibility of the author(s).

Conflicts of Interest: The authors declare no conflict of interest. The funders had no role in the design of the study; in the collection, analyses, or interpretation of data; in the writing of the manuscript; nor in the decision to publish the results.

\section{References}

1. Quintana, D.S.; Guastella, A.J.; Outhred, T.; Hickie, I.B.; Kemp, A.H. Heart rate variability is associated with emotion recognition: Direct evidence for a relationship between the autonomic nervous system and social cognition. Int. J. Psychophysiol. 2012, 86, 168-172. [CrossRef] [PubMed]

2. Nakasone, A.; Prendinger, H.; Ishizuka, M. Emotion recognition from electromyography and skin conductance. In Proceedings of the 5th International Workshop on Biosignal Interpretation, Tokyo, Japan, 6-8 September 2005; pp. 219-222.

3. Busso, C.; Deng, Z.; Yildirim, S.; Bulut, M.; Lee, C.M.; Kazemzadeh, A.; Lee, S.; Neumann, U.; Narayanan, S. Analysis of emotion recognition using facial expressions, speech and multimodal information. In Proceedings of the 6th international conference on Multimodal Interfaces, State College, PA, USA, 13-15 October 2004; ACM: New York, NY, USA, 2004; pp. 205-211.

4. Young, T.; Hazarika, D.; Poria, S.; Cambria, E. Recent trends in deep learning based natural language processing. IEEE Comput. Intell. Mag. 2018, 13, 55-75. [CrossRef] 
5. Mohammad, S.M.; Bravo-Marquez, F.; Salameh, M.; Kiritchenko, S. SemEval-2018 Task 1: Affect in Tweets. In Proceedings of the International Workshop on Semantic Evaluation (SemEval-2018), New Orleans, LA, USA, 5-6 June 2018.

6. Baziotis, C.; Athanasiou, N.; Chronopoulou, A.; Kolovou, A.; Paraskevopoulos, G.; Ellinas, N.; Narayanan, S.; Potamianos, A. NTUA-SLP at SemEval-2018 Task 1: Predicting Affective Content in Tweets with Deep Attentive RNNs and Transfer Learning. arXiv 2018, arXiv:1804.06658.

7. Kim, Y.; Lee, H.; Jung, K. AttnConvnet at SemEval-2018 Task 1: Attention-based Convolutional Neural Networks for Multi-label Emotion Classification. arXiv 2018, arXiv:1804.00831.

8. Gee, G.; Wang, E. psyML at SemEval-2018 Task 1: Transfer Learning for Sentiment and Emotion Analysis. In Proceedings of the 12th International Workshop on Semantic Evaluation, New Orleans, LA, USA, 5-6 June 2018; pp. 369-376.

9. Zhang, Y.; Wang, J.; Zhang, X. YNU-HPCC at SemEval-2018 Task 1: BiLSTM with Attention based Sentiment Analysis for Affect in Tweets. In Proceedings of the 12th International Workshop on Semantic Evaluation, New Orleans, LA, USA, 5-6 June 2018; pp. 273-278.

10. Meisheri, H.; Dey, L. TCS Research at SemEval-2018 Task 1: Learning Robust Representations using Multi-Attention Architecture. In Proceedings of the 12th International Workshop on Semantic Evaluation, New Orleans, LA, USA, 5-6 June 2018; pp. 291-299.

11. Karasalo, M.; Nilsson, M.; Rosell, M.; Bolin, U.W. FOI DSS at SemEval-2018 Task 1: Combining LSTM States, Embeddings, and Lexical Features for Affect Analysis. In Proceedings of the 12th International Workshop on Semantic Evaluation, New Orleans, LA, USA, 5-6 June 2018; pp. 109-115.

12. Rozental, A.; Fleischer, D. Amobee at SemEval-2018 Task 1: GRU Neural Network with a CNN Attention Mechanism for Sentiment Classification. arXiv 2018, arXiv:1804.04380.

13. González, J.Á.; Hurtado, L.F.; Pla, F. ELiRF-UPV at SemEval-2018 Tasks 1 and 3: Affect and Irony Detection in Tweets. In Proceedings of the 12th International Workshop on Semantic Evaluation, New Orleans, LA, USA, 5-6 June 2018; pp. 565-569.

14. Wang, W.; Chen, L.; Thirunarayan, K.; Sheth, A.P. Harnessing twitter "big data" for automatic emotion identification. In Proceedings of the 2012 International Conference on Privacy, Security, Risk and Trust (PASSAT) and 2012 International Conference on Social Computing (SocialCom), Amsterdam, The Netherlands, 3-5 September 2012; pp. 587-592.

15. Pak, A.; Paroubek, P. Twitter as a Corpus for Sentiment Analysis and Opinion Mining. In Proceedings of the LREC 2010, Valletta, Malta, 17-23 May 2010; Volume 10, pp. 1320-1326.

16. Nam, J.; Mencía, E.L.; Kim, H.J.; Fürnkranz, J. Maximizing Subset Accuracy with Recurrent Neural Networks in Multi-label Classification. In Proceedings of the Advances in Neural Information Processing Systems, Long Beach, CA, USA, 4-9 December 2017; pp. 5419-5429.

17. Mohammad, S.M.; Turney, P.D. Emotions evoked by common words and phrases: Using Mechanical Turk to create an emotion lexicon. In Proceedings of the NAACL HLT 2010 Workshop on Computational Approaches to Analysis and Generation of Emotion in Text, Los Angeles, CA, USA, 5 June 2010; pp. 26-34.

18. Mohammad, S.M.; Kiritchenko, S. Understanding Emotions: A Dataset of Tweets to Study Interactions between Affect Categories. In Proceedings of the 11th Edition of the Language Resources and Evaluation Conference, Miyazaki, Japan, 7-12 May 2018.

19. Landis, J.R.; Koch, G.G. The Measurement of Observer Agreement for Categorical Data. Biometrics 1977, 33, 159-174. [CrossRef] [PubMed]

20. Purver, M.; Battersby, S. Experimenting with distant supervision for emotion classification. In Proceedings of the 13th Conference of the European Chapter of the Association for Computational Linguistics, Avignon, France, 23-27 April 2012; pp. 482-491.

21. Ekman, P. Facial expression and emotion. Am. Psychol. 1993, 48, 384. [CrossRef] [PubMed]

22. Mohammad, S.M.; Turney, P.D. Crowdsourcing a word-Emotion association lexicon. Comput. Intell. 2013, 29, 436-465. [CrossRef]

23. Al-Kabi, M.N.; Al-Qwaqenah, A.A.; Gigieh, A.H.; Alsmearat, K.; Al-Ayyoub, M.; Alsmadi, I.M. Building a standard dataset for Arabie sentiment analysis: Identifying potential annotation pitfalls. In Proceedings of the 2016 IEEE/ACS 13th International Conference of Computer Systems and Applications (AICCSA), Agadir, Morocco, 29 November-2 December 2016; pp. 1-6. 
24. Roberts, K.; Roach, M.A.; Johnson, J.; Guthrie, J.; Harabagiu, S.M. EmpaTweet: Annotating and Detecting Emotions on Twitter. In Proceedings of the LREC, Istanbul, Turkey, 21-27 May 2012; Volume 12, pp. 3806-3813.

25. Fan, R.; Zhao, J.; Chen, Y.; Xu, K. Anger is more influential than joy: Sentiment correlation in Weibo. PLoS ONE 2014, 9, e110184. [CrossRef] [PubMed]

26. Bird, S.; Klein, E.; Loper, E. Natural Language Processing with Python: Analyzing Text with the Natural Language Toolkit; O'Reilly Media, Inc.: Sebastopol, CA, USA, 2009.

27. Albogamy, F.; Ramsay, A. Unsupervised Stemmer for Arabic Tweets. In Proceedings of the 2nd Workshop on Noisy User-Generated Text (WNUT), Osaka, Japan, 11 December 2016; pp. 78-84.

28. Albogamy, F.; Ramsay, A.; Ahmed, H. Arabic Tweets Treebanking and Parsing: A Bootstrapping Approach. In Proceedings of the Third Arabic Natural Language Processing Workshop, Valencia, Spain, 3-4 April 2017; pp. 94-99.

29. Fellbaum, C. WordNet; Wiley Online Library: Hoboken, NJ, USA, 1998.

30. Brill, E. Transformation-based error-driven learning and natural language processing: A case study in part-of-speech tagging. Comput. Linguist. 1995, 21, 543-565.

31. Badaro, G.; El Jundi, O.; Khaddaj, A.; Maarouf, A.; Kain, R.; Hajj, H.; El-Hajj, W. EMA at SemEval-2018 Task 1: Emotion Mining for Arabic. In Proceedings of the 12th International Workshop on Semantic Evaluation (SemEval-2018), New Orleans, LA, USA, 5-6 June 2018.

32. Soliman, A.B.; Eissa, K.; El-Beltagy, S.R. AraVec: A set of Arabic Word Embedding Models for use in Arabic NLP. Linguistics 2017, 1877, 0509. [CrossRef]

33. Park, J.H.; Xu, P.; Fung, P. PlusEmo2Vec at SemEval-2018 Task 1: Exploiting emotion knowledge from emoji and\# hashtags. arXiv 2018, arXiv:1804.08280.

34. De Bruyne, L.; De Clercq, O.; Hoste, V. LT3 at SemEval-2018 Task 1: A classifier chain to detect emotions in tweets. In Proceedings of the 12th International Workshop on Semantic Evaluation (SemEval-2018), New Orleans, LA, USA, 5-6 June 2018; pp. 123-127.

(C) 2019 by the authors. Licensee MDPI, Basel, Switzerland. This article is an open access article distributed under the terms and conditions of the Creative Commons Attribution (CC BY) license (http:/ / creativecommons.org/licenses/by/4.0/). 